seine Entwicklungslehre eine weitere Ausarbeitung des geistigen Prozesses, der sich im Leben des sich ausbildenden und entwickelnden Künstlers abspielt. Auch der Künstler ist (wie die Natur) in seinem. Können beschränkt, jeder hat nur sein ihm eigenes Talent (innere Gesetze), das ihm von Gott verliehen wurde. Dieses Talent kann er aber (wie die Natur) weiter entfalten, aber doch stets nur innerhalb der Grenzen seines Talents.

Das ist, kurz ausgedrückt, Goethes Stellung zum Entwicklungsgedanken, welche in dieser $\mathrm{Zu}$ sammenfassung wohl deutlicher hervortritt als in meinen historisch-kritischen Studien ${ }^{1}$, auf welche ich übrigens für die Beweisstellen und alle Details verweisen muß.

\section{Technische Bedeutung und neuere Darstellungsmethoden des Wasserstoffperoxyds.}

Von Dr. Max Wolf, Berlin-Charlottenburg.

Schon seit langer Zeit wird das Wasserstoffperoxyd $\left(\mathrm{H}_{2} \mathrm{O}_{2}\right)$ im großen Maßstabe dargestellt und findet vorteilhaft zu Oxydationszwecken in der Technik und auBerdem in der Medizin und Kosmetik Verwendung. Die Bedeutung der Oxy. dationsmittel im allgemeinen liegt darin, dab sie sogenannten ,aktiven" Sauerstoff enthalten, welcher viel wirksamer ist als der molekulare Sauerstoff der Luft; die Bedeutung des Wasserstoffperoxyds im besonderen aber ist in der Tatsache zu sehen, da $B$ kein anderer Stoff - prozentual gerechnet - so viel aktiven Sauerstoff gebunden enthält. Wasserstoffperoxyd ist daher der beste Sauerstoffakkumulator, denn auf $34 \mathrm{~g}$ lrommen $16 \mathrm{~g}$ aktiver Sauerstoff, d. h. $47 \%$, während $z$. B. beim Chlorkalk auf $127 \mathrm{~g} 16 \mathrm{~g}$ aktiver Sauerstoff zu rechnen sind. Diese Zahlen gelten aber nur für 100 prozentiges Wasserstoffperoxyd und für Chlorkalk von der theoretischen Zusammensetzung $\mathrm{CaCl}(\mathrm{OCl})$. Hinzu kommt noch, daß Wasserstoffperoxyd nach der Oxydationswirkung keine schädlichen Nebenprodukte hinterläBt, denn es zerfällt nach der Gleichung:

$$
\mathrm{H}_{2} \mathrm{O}_{2}=\mathrm{H}_{2} \mathrm{O}+\mathrm{O} \text {. }
$$

Man darf aber nicht verkennen, daß dem Peroxyd manche weniger günstige Eigenschaften anhaften, die seine Verwendungsmöglichkeit ziemlich stark einschränken. Abgesehen von dem im Verhältnis zu anderen Oxydationsmitteln ziemlich hohen Preise ist besonders zu beachten, daS die Hauptmenge des technisch dargestellten Produktes nur in Lösungen von $3-4$ Gew. $\% \mathrm{H}_{2} \mathrm{O}_{2}$ gewounen wird, wodurch insbesondere der Transport erheblich verteuert wird. Auch sind die rerdünnten Lösungen bei der Zersetzlichkeit des Peroxyds wenig haltbar.

1) Mistorisch-kritische Studien uber Goethe als Naturforscher. Würzburg 1912.
Es. gibt nun zwei Wege, diesen Ubelständen zu begegnen. Entweder man kann das Wasserstoffperoxyd konzentriertex in den Handel bringen (z. B. als 30 proz. Perhydrol), oder aber man führt es über in feste Verbindungen, in Persalze; so ist es gelungen, z. B. Perborate zu gewinnen, die bis $18 \%$ aktiven Sauerstoff, d. h. bis $40 \%$ Peroxyd enthielten. Immerhin muß man aber bedenken, daß die meisten dieser neuen Produkte, die therapeutischen und kosmetischen Zwecken dienen oder als sogenannte „Waschmittel" in den Handel gebracht werden (Pergenol, Clarax, Persil usw.), das Wasserstoffperoxyd niemals verdrängen werden, denn die große Mehrzahl derselben (auBer dem elektrolytisch gewonnenen Persulfat $\left[\mathrm{K}_{2} \mathrm{~S}_{2} \mathrm{O}_{8}\right]$ ) wird daraus dargestellt.

Bevor nun auf die Darstellungsmethoden näher eingegangen wird, sei noch einiges über die chemische Konstitution gesagt.

Das Wasserstoffperoxyd wurde im Jahre 1818 von Thénard entdeckt, als er Versuche anstellte über die Einwirkung versehiedener Säuren auf Bariumperoxyd, Thénard glaubte zunächst den Grundkörper für eine neue Klasse von Säuren gefunden zu haben, gelangte aber später zu der Auffassung, daß die von ihm entdeckte Verbindung ,oxydiertes Wasser" sei, also $\mathrm{H}_{2} \mathrm{O}+\mathrm{O}$, Im $\mathrm{Zu}$ sammenhange damit betrachtete man das eine Sauerstoffatom im Wasserstoffperoxyd als schwächer gebunden, und so erklärte sich das Oxydationsvermögen dieser Wasserstoff-Sauerstoff-Verbindung auf einfache Weise. Schwierigkeiten bereitete es aber, verständlich zu machen, warum Wasserstoffperoxyd auch reduzierend wirken kann, z. B. sind die Zersetzung von Kaliumpermanganat, die Abscheidung von Silber aus Silbernitratlösung solche Vorgänge. Man stelltè sich vor, dab das eine schwächer gebundene Sauerstoffatom des Wasserstoffperoxyds mit dem ,entgegengesetzt polarisierten" Sauerstoffatom des zu reduzierenden Körpers ein Molekül Sauerstoff $\mathrm{O}_{2}$ bildet. In dieser Anschauung ist schon die Antozontheorie Schönbeins, des berühmten Chemikers, enthalten, der der Nachwelt besonders durch die Entdeckung des Ozons bekannt geblieben ist.

Schönbein nahm an, dab Sauerstoff ,polarisiert" werden könne und nannte den negativen Ozon $\bar{O}$, den positiven Antozon $\stackrel{+}{0}$, ihre entsprechenden Verbindungen, die wir mit dem gemeinsamen Namen der Peroxyde oder Superoxyde zusammenfassen können, "Ozonide" und ,Antoozonide". Es würde in diesem Zusammenhange zu weit fuhren, wollte man auf die zahlreichen Untersuchungen und Argumente Schönbeins zur Stützung seiner Theorie näher eingehen. Hier genüge die Bemerkung, daß, wenngleich Schönbein mit zäher Energie bis an sein Lebensende (1868) seine Anschauung verteidigt hat, doch schon in den sechziger Jahren des vorigen Jahr. hunderts starke Zweifel geltend gemacht wurden. 
Im Jahre. 1870 hat dana Carl Engler unzweideutig nachgewiesen, daß Schönbeins Antozon und Wasserstoffperoxyd identisch sind.

Moritz Traube hat seit 1882 durch geistreiche und das Problem der Sauerstoffaktivierung erheblich fördernde Versuche die Fragen der Konstitution und der Bildung des Wasserstoffperoxyds wieder aufgenommen, und seine Anschauungen decken sich im wesentlichen auch mit den heute noch geltenden. Vor allem zeigte er, daß Peroxyd nicht durch Oxydation von Wasser entstehen kann, dap es also kein Oxydationsprodukt ist; es bildet sich vielmehr im Gegenteil durch einen Reduktionsvorgang, nämlich durch Einwirkung von ionisiertem Wasserstoff auf molekularen Sauerstoff gemäB der Gleichung:

$$
2 \mathrm{H} \cdot+\mathrm{O}_{2}=\mathrm{H}_{2} \mathrm{O}_{2} \text {. }
$$

Aus dieser Tatsache erklärt sich auch die Fähigkeit des Wasserstoffperoxyds, reduzierend zu wirken; denn - wie neuere Arbeiten ergeben haben - zerfallt die Verbindung in wässriger Lösung wieder in die Ionen $\mathrm{H}^{*}$ und $\mathrm{HIO}_{2}$. Diese freien H-Ionen können natürlich unter geeigneten Bedingungen reduzieren. Besonders entscheidend für die Auffassung Traubes waren die Resultate der Studien über die Autoxydation. Er konnte zeigen, daß bei der Autoxydation vieler Metalle, z. B. des Zinks, quantitativ Wasserstoffperoxyd gebildet wird. Als Reaktionsgleichung gilt:

$$
\begin{aligned}
& \mathrm{Zn}+2 \mathrm{H}_{2} \mathrm{O}=\mathrm{Zn}(\mathrm{OH})_{2}+2 \mathrm{H} \cdot \\
& 2 \mathrm{H} \cdot+\mathrm{O}_{2}=\mathrm{H}_{2} \mathrm{O}_{2} .
\end{aligned}
$$

Allerdings wirkt Zink dann wieder sekundär auf Wasserstoffperoxyd ein $\left(\mathrm{Zn}+\mathrm{H}_{2} \mathrm{O}_{2}=\right.$ $\left.\mathrm{Zn}(\mathrm{OH})_{2}\right)$, so dab eine quantitative Ausbeute nur erreicht werden kann, wenn man das Wasserstoffperoxyd im Augenblick des Entstehens bindet, also aus dem Reaktionsgemisch entfernt (etwa durch Baryt oder Kalk). Aus jenem Grunde entzieht sich die Bildung des Peroxyds auch meist der Beobachtung, und nur durch geschicktes Experimentieren gelingt es, dasselbe zu fassen.

Von neueren Autoren seien genannt Brühl, Spring, Haber, Manchot und vor allem Engler. Die Ansicht Englers kann man als Weiterführung des Traubeschen Gedankens bezeichnen. Auch nach seiner Auffassung ist die Bildung des Wasserstoffperoxyds ein Reduktionsvorgang, wobei angenommen wird, daß das Sauerstoffmoleküil ein ungesättigtes System darstellt, welches das Bestreben hat, in eine gesättigte Verbindung überzugehen. Man kann in diesem Zusammenhange die Bildung des Peroxyds direkt in Parallele stellen zu der von Sabatier beobachteten Addition von Wasserstoff an Aethylen mit Hilfe von Nickelpulver bei $300^{\circ}$.

oder

$$
\stackrel{\mathrm{CH}}{\mathrm{CH}}_{\mathrm{H}}^{\mathrm{CH}_{2}}+\underset{\mathrm{CH}}{\mathrm{H}}=\underset{\mathrm{CH}_{3}}{\mathrm{CH}_{3}}=\mathrm{C}_{2} \mathrm{H}_{6}
$$

$$
\stackrel{\mathrm{O}}{\mathrm{O}}+\underset{\mathrm{H}}{\mathrm{H}}=\underset{\mathrm{OH}}{\mathrm{OH}}=\mathrm{H}_{2} \mathrm{O}_{2} \text {. }
$$

Was num die Bildung und Darstellung des Wasserstoffperoxyds betrifft, so besteht das älteste Verfahren zur Gewinnung, nach dem auch heute noch der größte Teil des technischen Produktes erzielt wird, in der Zersetzung von Bariumperoxyd durch Säuren: z. B.

$$
\mathrm{Ba}<\mathrm{O}+\underset{\mathrm{H}}{\mathrm{O}}>\mathrm{SO}_{4}=\mathrm{BaSO}_{4}+\mathrm{H}_{2} \mathrm{O}_{2}
$$

Diese Reaktionsgleichung zeigt, wie sich an den Sauerstoff des Antozonides $\left(\mathrm{BaO}_{2}\right)$ die aus der Schwefelsäure stammenden Wasserstoffionen anlagern. An Stelle von Schwefelsäure hat man auch Salzsäure, Phosphorsäure, Kieselfluorwasserstoffsäure, Kohlensäure verwendet, an Stelle des Bariumperoxyds die Peroxyde der Alkalien oder der anderen Erdalkalien. Auf diesem Wege erhält man Lösungen von ca. $3-4$ Gew.\% Wasserstoffperoxyd. Dieselben lassen sich durch Konzentrieren auf dem Wasserbade, Destillieren im Vakuum, Extrahieren mit Aether (Wolffenstein) oder durch fraktionierte Kristallisation (Städel-Ahrle) schlieblich bis zu 100\%igem Wasserstoffperoxyd verarbeiten.

Das reine Produkt ist eine farblose, in dicker Schicht bläuliche, ölige Flüssigkeit, die sich in Ather löst. Sie ist bei Gegenwart auch nur geringer Mengen von Verunreinigungen leicht zersetzlich, sogar explosiv. Bei tiefer Temperatur erstarrt sie zu schön ausgebildeten säulenförmigen Kristallen.

Aus Persãuren ođer deren Salzen erhält man mit Wasser oder einer Säure Lösungen von Wasserstoffperoxyd. So ist der Firma Merel in Darmstadt vor einigen Jahren ein Verfahren patentiert worden, wonach aus Bariumperkarbonat durch Einwirkung von Wasser oder einer Säure Wasserstoffperoxyd gebildet wird, im Sinne folgender Gleichungen:

oder

$$
\mathrm{BaCO}_{4}+\mathrm{H}_{2} \mathrm{O}=\mathrm{BaCO}_{3}+\mathrm{H}_{2} \mathrm{O}_{2}
$$

$$
\mathrm{BaCO}_{4}+\mathrm{H}_{2} \mathrm{SO}_{4}=\mathrm{BaSO}_{4}+\mathrm{H}_{2} \mathrm{O}_{2}+\mathrm{CO}_{2} .
$$

Sehr gute Resultate soll auch im Großbetriebe das Persulfatverfahren von Adolph und Pietsch geliefert haben. Dasselbe beruht auf der Umsetzung von elektrolytisch gewonnenem Persulfat in Carosche Saure $\mathrm{H}_{2} \mathrm{SO}_{5}$ und auf der Spaltung dieser in Wasserstoffperoxyd ind Schwefelsäure im Sinne der Gleichungen:

$$
\begin{aligned}
\mathrm{K}_{2} \mathrm{~S}_{2} \mathrm{O}_{3}+\mathrm{H}_{2} \mathrm{SO}_{4} & =\mathrm{K}_{2} \mathrm{~S}_{2} \mathrm{O}_{7}+\mathrm{H}_{2} \mathrm{SO}_{5} \\
\mathrm{H}_{2} \mathrm{SO}_{5}+\mathrm{H}_{2} \mathrm{O} & =\mathrm{H}_{2} \mathrm{SO}_{4}+\mathrm{H}_{2} \mathrm{O}_{2}, \\
\mathrm{~K}_{2} \mathrm{~S}_{2} \mathrm{O}_{7}+\mathrm{H}_{2} \mathrm{O} & =2 \mathrm{KHSO}_{4} .
\end{aligned}
$$

Das Wasserstoffperoxyd wird abdestilliert, und die Bisulfatlauge kann wieder zur elektrolytischen Darstellung des Persulfats dienen; so ergibt sich ein KreisprozeB, in dem eigentlich nur elektrische Energie verbraucht wird.

Die bisher besprochenen Verfahren beruhten sämtlich auf rein chemischen Umsetzungen und Spaltungen ron Peroxyden and Persalzen, es sei nun eingegangen auf diejenigen, die zu ihrer Aus- 
führung hoher Temperaturen odex elektrischer Energie bedürfen.

Die Bildung von Wassexstoffperoxyd kann je nach den Bedingungen, von denen man ausgeht, sowohl als ein endothermer wie auch als ein exothermer Vorgang aufgefaBt werden. Betrachtet man die Bildung aus Wasser und Sauerstoff, so liegt ein endothermer Prozel vor, denn die Reaktion verläuft nach der Gleichung:

$$
\mathrm{H}_{2} \mathrm{O}+\frac{\mathrm{O}_{2}}{2}=\mathrm{H}_{2} \mathrm{O}_{2}-22160 \mathrm{cal} \text {. }
$$

Geht man aber von den Elementen aus, d. h. von Wasserstoff und Sauerstoff, so stellt sich die Wasserstoffperoxyd-Bildung als ein exothermer Vorgang dar:

$$
\mathrm{H}_{2}+\mathrm{O}_{2}=\mathrm{H}_{2} \mathrm{O}_{2}+46840 \text { cal. }
$$

Aus diesen beiden Gleichungen ergibt sich ohne weiteres, dab die erste Reaktion nur unter Wämeabsorption vor sich gehen kann. Will man also aus Wasser plus Sauerstoff Wasserstoffperoxyd erhalten, so muß man Wärme zuführen, d. h. man muB bei hoher Temperatur arbeiten. Man kann aber aus einem gegebenen Volumen Wasser und Sauerstoff nur eine ganz bestimmte, durch die Temperatur beeinflußte Ausbeute an Wasserstoffperoxyd erhalten. Es stellt sich nämlich schnell ein Gleichgewicht ein :

$$
\left.\mathrm{H}_{2} \mathrm{O}+\mathrm{O} \underset{\mathrm{H}}{\leftarrow} \mathrm{H}_{2} \mathrm{O}_{2}{ }^{1}\right)
$$

das sich mit steigender Temperatur immer mehr nach rechts verschiebt, wie Arbeiten von Nernst ergeben haben. Aber bei so hoher Temperatur ist auch die Zerfallsgeschwindigkeit sehr groß, und deswegen ist rapide Abkühlung der erhitzten Gase auf einen Temperaturbereich mit geringer Zerfallsgeschwindigkeit dringend notwendig, wenn man die im Gleichgewicht gebildete Menge Peroxyd gewinnen will.

Nach diesem Prinzip hat man die Bildung von Wasserstoffperoxyd mehrfach herbeiführen können. Traube hat zuerst gezeigt, daß, wenn man eine Wasserstofflamme auf Wasser richtet, d. h. also die Verbrennungsgase plötzlich abkühlt, Wasserstoffperoxyd entsteht. Neuere Autoren auf diesem Gebiete sind Franz Fischer und seine Mitarbeiter. Wenn man Wasserdampf oder Wasserdampf plus Sauerstoff gegen eine Wasserstoffflamme blies, durch glühende Röhrchen preßte, usw., wenn man unexplosible Gemische von Wasserstoff und Sauerstoff über glühende Drähte leitete, und die Reaktionsprodukte schnell abkühlte, ergaben sich stets wasserstoffperoxydhaltige Lösungen. Auf der gleichen Erscheinung beruht ein Patent der Firma $G$. A. F. Kahlbaum, Berlin, welches dadurch gekennzeichnet ist, daB wasserdampfhaltige Gase oder Wasserdampf allein

1) Diese Gleichung deutet scheinbar im Widerspruch zu dem oben Gesagten eine Oxydation von Wasser an. Man muB aber bedenken, daß bei der hohen Temperatur ein Teil des Wassers dissozifert ist, so dab tatsächhich die Reaktion zwischen den Elementen vor sich geht. und Heizquellen, wie elektrische Flammenbögen, glühende Nernststifte, Funkenstrecken usw. gegeneinander mit einer Geschwindigkeit ron mindestens $1 \mathrm{~m}$ pro Sekunde bewegt werden.

Für die technische Verwertung aber dürften all diese Methoden unbrauchbar sein, hauptsächlich weil wegen der Gegenwart von Wasserdampf immer nur sehr verdünnte Lösungen erhalten werden können. Viel mehr Erfolg von diesem Gesichtspunkt aus versprechen die elektrischen Methoden, besonders auch deswegen, weil man nach diesen das Wasserstoffperoxyd in größerer Konzentration erhalten kann.

Es ist oben gesagt worden, die Bildung von Wasserstoffperoxyd geschähe durch Anlagerung von Wasserstoffionen an molekularen Sauerstoff (2 $\mathrm{H} \cdot+\mathrm{O}_{2}=\mathrm{H}_{2} \mathrm{O}_{2}$ ). Ein billiges und bequemes Mittel, ionisierten Wasserstoff $\mathrm{zu}$ erzielen, ist die Elektrolyse, und so hat schon Traube, als er an die Kathode eines Elementes Sauerstoff leitete, mit quantitativer Ausbeute: Wasserstoffperoxyd erhalten können. Diese Versuche haben in neuester Zeit Franz Eischer und Otto Prieß wieder aufgenommen ${ }^{1}$ ). Durch Anwendung von sehr groben Sauerstoffdrucken und durch starkes Rühren des Elektrolyten während des Stromdurchganges ist es ihnen gelungen, ein Verfahren auszuarbeiten, welches technisch sehr wertvoll werden durfte, welches vielleicht sogar berufen ist, den alten ProzeB der. Zersetzung von Bariumperoxyd zu verdrängen; denn die Strom ausbeute betrug bei 100 at. Druck ca. 300-400 g Wasserstoffperoxyd pro K. W. St. Auf diesen Prinzipien beruht ein Patent, welches erst kürzlich der Firma Henckel \& Co. in Düsseldorf erteilt worden ist, wonach man durch kathodische Reduktion von Sauerstoff unter hohem Druck kontinuierlich Wasserstoffperoxyd gewinnt.

Durch Elektrolyse von borsäurehaltigren Lösungen mit überlagertem Wechselstrom hat Ennst Bürgin2) Wasserstoffperoxyd anodisch erhalten. Nach seiner Auffassung geht die Bildung im. Sinne folgender Gleichung vor sich:

$$
2 \mathrm{OH}^{\prime \prime}+2 \mathrm{O}=\mathrm{H}_{2} \mathrm{O}_{2} \text {. }
$$

$60 \%$

Die Stromausbeute betrug an Zinkanoden

Die stille elektrische Entladung hat sich mehrfach als ein ausgezeichnetes Mittel erwiesen, elektrische Energie in feiner Verteilung und so wenig heftig auf Gase wirken zu lassen, daß die Reaktionsprodukte nicht sogleich einem sekundären Zerstörungsprozeß erliegen. Auf dieser Erfahrung basieren die Verfahren von Alexandre de Hemptinne) and unabhängig davon von Fr. Fischer und Max Wolfs), welche dadurch gekenn-

1) Fischer und Pries, Ber. d. D. Chem. Ges. 46, 698 (1913).

2) Bürgin, Beiträge zur Kenntnis der Entstehung von Perboraten durch Elektrolyse. Diss. Berlin 1911. 3) Hemptinne, Ann. de la Sociête scientifique de Bruxelles, 1911.

4) Wolf, Zeitschr. f. Elektrochemie, 20, 204 (1914).* 
zeichnet sind, dal unexplosible Gemenge von Wasserstoff und Sauerstoff mit WasserstoffüberschuB der stillen elektrischen Entladung ausgesetzt werden. Diese bewirkt offenbar durch Ionenstob die Dissoziation der Wasserstoffmolekule. Sekundär findet dann die Anlagerung dieser Atome an Sanerstoffmoleküle, d. h. die Bildung von Wasserstoffperoxyd statt. Die Arbeitsweise Hemptinnes ist nun derart, daB er das Gasgemisch aus einem Gasometer durch eine Berthelotsche Röhre und dann zur Absorption des gebildeten Wasserstoffperoxyds durch Wasser im Kreislauf leitet. Auf diesem Wege kann er natürlich niemals zu hochkonzentriertem Produkt gelangen. Aus seinen Zahlen Iäßt sich berechnen, daß er Lösungen von 0,08. Gew.-.\% Wasserstoffperoxyd erhalten hat.

Dagegen ist es Fischer und Wolf gelungen, unter Einhaltung genau studierter Versuchsbedingungen 100 proz. Peroxyd zu gewinnen. Das Produkt wurde durch tiefe Temperatur in der Berthelotschen Röhre kondensiert. Dieses Verfahren hat den großen Vorzug, daß man bei Anwendung reiner Gase durch direkte Synthese ein absolut reines Produkt herstellen kann. Allerdings sind die Stromkosten ziemlich hoch, und es bleibt abzuwarten, ob es möglich sein wird, dieselben so weit herabzusetzen, dab das Verfahren konkurrenzfähig wird.

Mögen diese kurzen Ausführungen gezeigt haben, mit welchem Eifer und in welchem Sinne Wissenschaft und Technik an dem Problem arbeiten, die wertvollen Eigenschaften des Wasserstoffperoxyds allgemein nutzbar zu machen.

\section{Über einige scheinbare physikalisch- chemische Anomalien.}

Von Dr. F. Marshall, Halle a. S.

Nachdem kürzlich das schöne Werk von Weinbergs „Kinetische Stereochemie der Kohlenstoffverbindungen"1) erschienen ist, fällt es an der Hand der kinetischen Theorien nicht schwer, auch einige scheinbare Widersprüche auf anorganischem Gebiete aufzuklären, ohne auf stereochemische Verhältnisse selbst näher einzugehen.

1. Ofters ist zum Beispiel bereits die Frage aufgeworfen worden: wie kommt es, daß Wasser, welches aus zwei gasförmigen Komponenten gebildet wird, flüssig ist, während der nahe verwandte Schwefelwasserstoff, der aus einem gasförmigen und einem festen Elemente gebildet wird, selbst gasförmig ist. Wie ist es ferner zu erklären, dab bei der völligen Unschädlichkeit des als Nahrungsmittel für Tiere und Pflanzen unentbehrlichen Wassers das Schwefelwasserstofigas ein gefährliches Gift ist? In diese. Verhältnisse vermag nun die kinetische Anschauungsweise Licht $\mathrm{zu}$ brin-

1) von Weinberg, "Tinetische Stereochemie der Kohlenstoffverbinduagen". Braunschweig, Fr. Vieweg u. Sohn, 1914. gen. Die Atome eines jeden Elementes enthalten einen Betrag von Energie und zwar Bewegungsenergie, bei der Vereinigung von Atomen verschiedener Elemente pflegt nun in der Mehrzah! der Fälle diese Bewegungsenergie der beteiligten Atome um einen bestimmten Betrag vermindert zu werden, und zwar wird sie in Form von Wärme, der sogenannten Bildungswärme der betreffenden Verbindung, abgegeben: Je größer nun diese Bildungswärme ist, um so geringer wird die Energiesumme sein, die in der Verbindung der Atome zurückbleibt, d. h. mit anderen Worten die Beweglichkeit der Atome. Umgekehrt, je geringer die Bildungswärme, desto größere Beweglichkeit verbleibt den verbundenen Atomen. MuB man endlich gar, wie in einer Anzahl von Fällen (endothermische Verbindungen) noch Energie zuführen, um eine Vereinigung von Atomen verschiedener Elemente zu erzielen, so ist die Beweglichkeit der Einzelatome so groß, daß sie zu plötzlicher explosionsartiger Zersetzung der Verbindung führen kann.

Aus einem Vergleich der Bildungswärmen können wir nun ohne weiteres folgern, daß im Wasser $(68,3 \mathrm{Kal}$.) die Einzelatome weit weniger beweglich sind als im Schwefelwasserstoff $(4,6$ bzw. 2,8 Kal.), was des weiteren auch durch die viel größere Beständigkeit des Wassermoleküls gegenüber dem leicht zersetzbaren Schwefelwasserstoffmolekül bewiesen wird. - Daß die Beweglichkeit der Atome einen starken Einflub auf den Aggregatzustand des Moleküls hat, ist ohne weiteres klar. Durch Erwärmen verstärken wir bekanntlich den Bewegungsgrad von Atomen und benutzen diese Tatsache auch vielfach, um Reaktionen zu erleichtern, durch Erwärmen vermögen wir aber zugleich feste Körper zu schmelzen und zu vergasen. Weitere Beweise für die relative Starrheit des Wassermoleküls im Vergleich zum Schwefelwasserstoffmolekül sind zunächst die erst bei $1000^{\circ}$ beginnende Dissoziation des Wassers, während Schwefelwasserstoff schon bei gewöhnlicher Temperatur allmählich zersetzt wird. Ferner gelingt die Bildung von Wasserstoffperoxyd nur unter Energiezufuhr, während Schwefelwasserstoff verhältnismäßig leicht 1-3 Atome Schwefel addiert. Die Beständigkeit dieser Wasserstoffsupersulfide läßt sich beträchtlich erhöhen, wenn man in ihnen die Atombewegungen hemmt, also eine Belastung im von Weinbergschen Sinne herbeiführt, so existiert eine kristallisierte Verbindung von Strychnin mit Wasserstofftrisulfid und eine solche von Bruzin mit Wasserstoffdisulfid. Endlich wäre noch in Betracht zu ziehen, daB das Wasser ein Verbrennungsprodukt ist, während beide Komponenten des Schwefelwasserstoffs noch brennbar sind, d. h. also noch thre ganze Verbrennungswärme abzugeben vermögen.

Die Giftigkeit des Schwefelwasserstoffes muB gleichfalls als eine Folge des lebhaften Bewegungszustandes angesehen werden, dafür ist zwar 